Rabaska

Revue d'ethnologie de l'Amérique française

\title{
Le vol des soupes aux choux : une tradition acadienne de Pombcoup?
}

\section{The theft of cabbage soup: an Acadian tradition from Pubnico ?}

\section{Carmen d'Entremont}

Volume 6, 2008

URI : https://id.erudit.org/iderudit/019981ar

DOI : https://doi.org/10.7202/019981ar

Aller au sommaire du numéro

Éditeur(s)

Société québécoise d'ethnologie

ISSN

1703-7433 (imprimé)

1916-7350 (numérique)

Découvrir la revue

Citer cet article

d'Entremont, C. (2008). Le vol des soupes aux choux : une tradition acadienne de Pombcoup ? Rabaska, 6, 69-78. https://doi.org/10.7202/019981ar
Résumé de l'article

En Acadie, pendant la première moitié $\mathrm{du} \mathrm{xx}^{\mathrm{e}}$ siècle, les tours étaient un amusement commun parmi les jeunes garçons. Se limitant surtout à des exemples et des résultats tirés d'une étude récente sur la tradition orale de Pubnico, Nouvelle-Écosse, cette note de recherche présente une mystification particulière qui fut en pleine vitalité dans ce village acadien au début $d u x^{e}$ siècle : le vol des soupes aux choux. Ce rituel d'automne se déroulait ordinairement en soirée, dans le cadre d'une veillée organisée. Les hôtes invitaient quelques amis à venir jouer aux cartes, chanter et, bien sûr, manger de la soupe. En plus des attraits de la rencontre sociale, on voulait voir si la soupe serait volée. Selon plusieurs attestations, le but était d'emporter la soupe dans un autre lieu dans l'intention de la consommer avant d'être découvert, car les victimes circulaient souvent d'une maison à l'autre en espérant retrouver leur soupe. Après avoir confirmé la présence de cette tradition unique dans ce milieu, la question posée est de savoir si le vol des soupes aux choux est une coutume née localement, ou si on l'a vécue ailleurs. 


\title{
Le vol des soupes aux choux : une tradition acadienne de Pombcoup?
}

\author{
CARMEN D'ENTREMONT \\ Université Sainte-Anne
}

Avant l'arrivée de la télévision, des jeux vidéo et, plus récemment, de l'Internet, les jeunes Acadiens s'amusaient à divers jeux. Certains, plus malicieux, aimaient passer leur temps à mystifier la population. On associe notamment cette pratique à l'automne, surtout à l'Halloween, temps désigné pour jouer des tours. Comme le souligne l'ethnologue Jean-Claude Dupont, « on appelait souvent le 31 [octobre] au soir le "soir des tours" ». À Pubnico, on parle plutôt de trick time: "Trick time, c'était l'automne, c'était la Hallowe'en ${ }^{2}$ ». Partout en Acadie on déplaçait des objets, on démontait des charrettes pour les mettre sur le toit des granges, on chavirait les «petites maisons » [toilettes extérieures], on peinturait les animaux de la ferme et on volait les légumes des jardins, surtout les choux, «car la coutume voulait que le soir de la Toussaint on y mangeât de la soupe aux choux ${ }^{3} »$. À Pubnico, ce mets était plutôt consommé la veille de la Toussaint, le 31 octobre. Et, en plus de prendre les légumes, on volait carrément les soupes. Une enquête menée entre 2004 et 2006 révèle que la coutume du vol des soupes aux choux fut autrefois une coutume en pleine vitalité à Pubnico, en Nouvelle-Écosse, mais moins connue ailleurs. Maintenant que je peux confirmer la présence de cette pratique dans ce milieu, la question que je me pose est de savoir si le vol des soupes aux choux est une coutume née localement, ou si on l'a vécue ailleurs.

Cet exposé se limite surtout à des exemples et des résultats tirés d'une étude récente sur la tradition orale de Pubnico-Ouest, mon village d'origine. Dans le cadre de mon projet de maîtrise, j'avais mené un important travail de terrain: il s'agissait d'évaluer le potentiel folklorique de cette région

1. Jean-Claude Dupont, Héritage de la francophonie canadienne : tradition orale, Sainte-Foy, Les Presses de l’Université Laval, 1986, p. 52.

2. Centre acadien (CA), Université Sainte-Anne (Nouvelle-Écosse), coll. Carmen d'Entremont, témoignage de M. Florent d'Entremont (né le 24 septembre 1935), enregistré le 7 février 2005 à PubnicoOuest-le-Bas.

3. Dupont, op. cit., p. 53. 
acadienne $^{4}$. L'enquête, qui révèle que la pratique des récits oraux est un phénomène culturel encore dynamique dans ce milieu, met en valeur l'importance de récits appartenant à des genres moins classiques qui intéressaient peu les chercheurs. Plus d'une centaine de textes ont été transcrits et classés. Le répertoire, constitué d'une vingtaine d'heures d'enregistrements effectués sur une période d'environ deux ans, contient des contes, des récits légendaires, des blagues, des histoires vraies et des mystifications. Comme la tradition des tours avait été si vigoureuse dans cette communauté, je lui avais réservé une section entière : elle contient vingt-trois récits de tours, dont six portent sur le vol des soupes.

Mon intérêt pour la mystification s'est éveillé pendant une enquête dans le cadre du cours Littérature orale I : le conte populaire et la légende, dispensé à l'Université Sainte-Anne par l'ethnologue Jean-Pierre Pichette. Pour ce travail, j'avais interrogé un ami de la famille, Florent d'Entremont, pour qui les tours d'autrefois sont des souvenirs qu'il chérit encore aujourd'hui. Né en 1935, il a été témoin de plusieurs mystifications et m'a transmis des attestations précieuses sur les tours joués dans son village. L'année précédente, j'avais aussi recueilli quelques récits de tours pour une étude sur les coutumes d'autrefois sous la direction du professeur Barry-Jean Ancelet à l'Université de la Louisiane à Lafayette. C'est à ce moment que j'appris l'existence de la fameuse pratique du vol de la soupe aux choux. Ma recherche initiale sur la mystification et sur le vol des soupes commençait donc avec ces deux premières expériences de terrain. En poursuivant ma recherche, j'ai réalisé que la majorité des gens de Pubnico-Ouest, nés pendant la première moitié $\mathrm{du} \mathrm{XX}^{\mathrm{e}}$ siècle, connaissaient cette mystification particulière.

Durant les étés 2005 et 2006, mon insertion dans l'équipe du Laboratoire de littérature orale (LABOR), au Centre acadien de l'Université Sainte-Anne, m'a permis d'élargir ma recherche et de sonder, avec mes collègues, la région du Sud-Ouest (Pubnico, l'Île-des-Surette, Wedgeport et plusieurs villages de la baie Sainte-Marie) et de l'Île-du-Cap-Breton (l'Île-Madame, Chéticamp et Saint-Joseph-du-Moine). Ces enquêtes ont révélé qu'on avait volé un bassin de râpure à la baie Sainte-Marie et du fricot à Grand-Étang, dans la paroisse de Saint-Joseph-du-Moine, deux mets traditionnels des Acadiens, ainsi que du fudge [sucrerie] dans ces deux milieux ${ }^{5}$. S'il est possible qu'on trouve des parallèles ailleurs, nous n'avons toutefois pu recueillir aucune attestation d'un vol de soupe aux choux en dehors du village de Pubnico-Ouest.

Le travail de terrain, la discussion avec des ethnologues et des informateurs d'un peu partout, et la consultation des ouvrages disponibles

4. Carmen d'Entremont, «Contes, légendes, histoires et mystifications : la tradition orale de Pubnico-Ouest », Mémoire de maîtrise, University of Louisiana at Lafayette, 2006, viii-340 p.

5. CA, coll. LABOR-Gaudet-Thériault et coll. LABOR-d'Entremont-Dol. 
indiqueraient que le vol des soupes aux choux à Pubnico-Ouest est une tradition unique, s'étendant probablement à quelques villages avoisinants. Aussi est-il important de documenter cette coutume pendant qu'elle reste vivante dans la mémoire des villageois qui l'ont vécue, car sa pratique se serait graduellement éteinte vers le milieu du $\mathrm{Xx}^{\mathrm{e}}$ siècle. D'où cette note de recherche.

\section{Le village de Pombcoup / Pubnico-Ouest}

Fondé en 1653, Pubnico est un petit village de pêche qui comprend une population d'environ 2000 âmes. Il fait partie de la municipalité d'Argyle, qui regroupe plus d'une douzaine de villages francophones situés, pour la majorité, le long des côtes de l'extrémité sud-ouest de la Nouvelle-Écosse. Pubnico porta d'abord un nom micmac que les Acadiens prononceront Pobomcoup et Pombcoup jusqu'à ce que le nom prenne officiellement la forme anglaise de Pubnico. De nos jours, on fait souvent référence aux « Pubnicos » puisqu'une série de villages portant des variations du même nom entourent le havre de Pubnico, la population du côté est étant surtout anglophone tandis que le côté ouest est constitué majoritairement d'Acadiens. Ceux-ci furent parmi les derniers déportés vers les colonies anglo-américaines (1756-1758), mais parmi les premiers à revenir (1766). Au retour, une vingtaine de familles s'installèrent de chaque côté du havre, près de leur

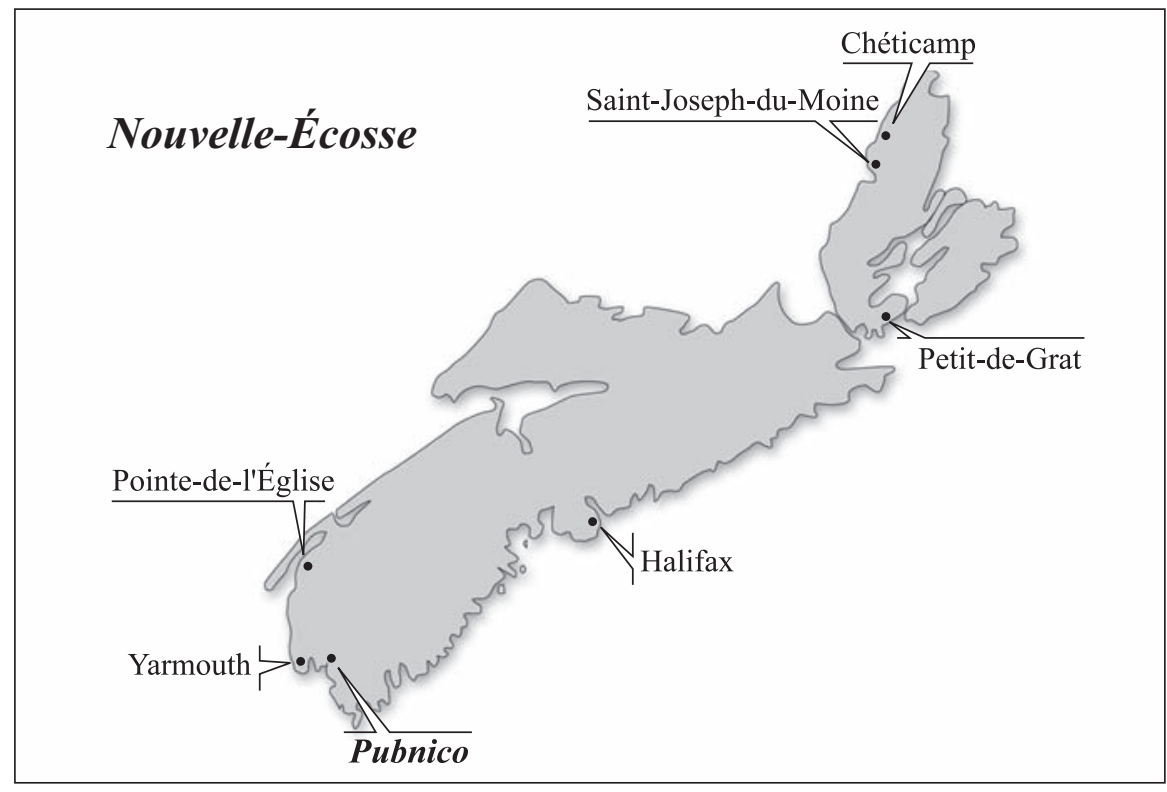

La Nouvelle-Écosse.

Carte préparée par Jason Saulnier, Centre acadien, Pointe-de-l’Église. 
ancienne baronnie, sur les terres vierges que les Anglais n'avaient pas encore touchées : ce fut le deuxième établissement de Pubnico. Fondé au milieu du $\mathrm{XVII}^{\mathrm{e}}$ siècle, Pubnico est considéré comme le premier village encore occupé par des Acadiens, ainsi que le plus ancien village du Canada toujours habité par les descendants de son fondateur. Longtemps restés isolés, ces derniers ont conservé une identité assez unique.

Puisque les gens de Pubnico se connaissaient bien, ils se rassemblaient souvent pour accomplir des travaux collectifs, surtout à la ferme, mais aussi pour se divertir, partager un repas, échanger des nouvelles, jouer aux cartes et chanter entre eux. Les maisons qui possédaient un orgue, comme celles où évoluaient des joueurs d'accordéon et de violon, étaient populaires. Toutefois, l'amour de la musique, du chant et de la danse ne fut pas la seule raison d'organiser ces veillées. C'était la pratique de servir un goûter, surtout de la soupe aux choux, à ces soirées d'automne. Pour les jeunes célibataires, c'était une occasion idéale pour les fréquentations. Aussi, les jeunes profitaient-ils des rassemblements pour jouer des tours, tels que voler la soupe, comme l'explique cette informatrice : «Si t'avais le poté de soupe sur le stove [poêle], les jeunes auriont essayé à venir voler la soupe, tu sais ${ }^{6} »$.

Vers la fin des années 1920, avec l'arrivée de la radio, des journaux et, plus tard, de la télévision, on a commencé à voir l'impact des nouveaux médias. Éventuellement, ces divertissements nouveaux remplacèrent les activités du passé et espacèrent les rassemblements. La vie traditionnelle sur les petites fermes familiales, qui caractérisait le village de mes ancêtres, est donc en régression depuis la deuxième moitié $\mathrm{du} \mathrm{Xx}^{\mathrm{e}}$ siècle, de sorte que les coutumes du temps de mes grands-parents et de mes parents sont disparues, y compris la fameuse pratique du vol des soupes aux choux. Heureusement, ceux qui ont vécu pendant ces années n'ont pas oublié la ferme familiale et plusieurs gardent de forts et clairs souvenirs de cette belle tradition.

\section{La mystification et le « soir des tours » en Acadie}

Même si on mystifiait à l'année longue en Acadie, le plus grand nombre de tours, au moins les plus célèbres, semblent avoir eu lieu pendant la période automnale, jusqu'à la veille de la Toussaint. Le 31 octobre, à l'Halloween, lorsque les filles défilaient devant les fenêtres en portant leurs « faces de citrouilles $^{7} »$, les garçons passaient leur temps à jouer des tours. D'après le

6. CA, coll. Carmen d'Entremont, témoignage de $\mathrm{M}^{\mathrm{me}}$ Marjorie d'Entremont (née le 13 septembre 1917), enregistré le 24 novembre 2004 à Pubnico-Ouest-le-Bas.

7. Autrefois, à l'Halloween, les filles évidaient leurs citrouilles pour sculpter des visages et mettre des chandelles à l'intérieur, car c'était la coutume de passer de maison en maison, devant les fenêtres, pour que les gens du village, surtout les aînés, puissent voir leurs créations. On nommait ces citrouilles allumées « faces de citrouilles ». 
Petit Robert, la mystification est un acte ou propos destiné à tromper un individu en abusant de sa crédulité et pour s'amuser à ses dépens. À Pubnico, on l'appelle familièrement trick, et si on a réussi à mystifier un individu, on dit qu'on l'a «attrapé ». En plus d'être un amusement, la mystification représente une coutume, une manière de vivre ; elle fait partie de l'identité acadienne depuis longtemps. Dès le début de la colonie, nos ancêtres eurent recours à diverses ruses, par exemple, pour déjouer des visiteurs non voulus tels que les pirates ${ }^{8}$.

Certes, pendant la première moitié $\mathrm{du} \mathrm{Xx}^{\mathrm{e}}$ siècle, les tours étaient un amusement commun parmi les jeunes garçons. Pour plusieurs, c'était le seul amusement, comme le souligne cet informateur : « c'est tout ce [qu']on avait à faire ${ }^{9} »$. Souvent, quelques-uns d'entre eux se rencontraient à la nuit tombante dans l'intention de mystifier les gens du milieu. Parfois, on avait déjà exécuté un plan pendant le jour à l'école comme le raconte cet informateur : "J'étions une gang de jeunes là que, about la même âge, à l'école au même temps. Et ça planait le jour pour aller le soir ${ }^{10} »$. Mais ordinairement, les tours étaient planifiés spontanément lors de ces rencontres. D'habitude, on choisissait de duper un voisin. On visait surtout à les faire sursauter et enrager. En général, les tours étaient joués sans malice, mais les jeunes pouvaient se venger de ceux qui leur avaient fait du tort et de ceux qui s'en fâchaient. Si une victime s'irritait facilement et qu'elle poursuivait les coupables, c'était un plaisir pour eux, car une bonne poursuite, c'était une réussite ; comme le souligne cet informateur: " Si t'avais point une chase, ça valait point $m u c h$ la peine que tu fus [fusses : d'y avoir été] ${ }^{11} \gg$ ou encore : «Il y avait quelques familles qui s'enrageaient, puis les jeunes aimaient surtout aller à ces maisons-là. Si personne réagissait à leur trick, bien ils sentaient qu'ils avaient perdu leur temps, bien sûr ${ }^{12}$ ». C'était aussi le cas à Chéticamp, comme le souligne une informatrice : "S'ils savaient qu'il y en avait un qu'était malin, là, ou dans le sens qu'il s'enrageait facilement, c'était sûr que lui, ils alliont y jouer des tours ${ }^{13} »$. On dit qu'à Pubnico une dame lançait des seaux d'eau aux ratoureux ; d'autres les chassaient à coups de fusil. D'habitude les tours n'étaient pas pris au sérieux et les victimes riaient de la situation, tout en préparant leur revanche. On ne voulait surtout pas détruire des biens

8. Désiré d'Eon, Histoires de chez-nous : faits et anecdotes d'un temps qui n'est plus, Yarmouth, L'imprimerie Lesbarbot, 1977, p. 38-46.

9. CA, coll. Carmen d'Entremont, M. Florent d'Entremont, le 7 février 2005.

10. Loc. cit.

11. Loc. cit.

12. CA, coll. LABOR-d'Entremont-Dol, témoignage de $\mathrm{M}^{\text {me }}$ Imelda Currans (née le 22 septembre 1931), enregistré le 27 juillet 2006 à Pubnico-Ouest.

13. CA, coll. LABOR-d'Entremont-Dol, témoignage de $\mathrm{M}^{\mathrm{me}}$ Marie-Colombe Robichaud (née le 4 octobre 1942), enregistré le 19 juillet 2006 à Meteghan-le-Centre. 
ou faire du mal à personne; l'on mystifiait pour s'amuser et pour le plaisir de faire marcher quelqu'un : " Ils défaisiont point beaucoup de butin. C'était rien que pour faire un trick. [...]. C'était rien que pour s'amuser, ouais. T'avais point [de cas] où [est-]ce qu'ils auriont brûlé le butin ${ }^{14} \gg$. Mais les accidents étaient possibles : par exemple, des jeunes farceurs renversèrent une écrémeuse remplie de lait lors de l'exécution d'un tour, et un autre groupe endommagea, sans le vouloir, un poêle de cuisine en roulant une grosse citrouille dans la maison. Dans les deux cas, les auteurs furent vraiment déçus du résultat, ce qui est représentatif du respect qu'on avait envers les autres membres de la communauté.

En plus de déplacer et de cacher les objets des autres, on allait souvent voler les légumes des jardins tels que les navots [navets] et les choux. À Pubnico, c'était souvent dans l'intention de les lancer par la porte des maisons pour enrager les autres, et les faire sortir. Comme le mentionne cette informatrice : «On allait voler les navots et les choux hors des petits jardins. Et on rouvrait les portes des maisons, on les tirait dedans ${ }^{15} »$. D'autres les prenaient pour faire une soupe aux choux, ce qu'on nomme aussi Halloween soup, et rassembler les gens de son voisinage. Mais, comme le souligne une informatrice, "ils disiont jamais à qui c'était les choux ${ }^{16}$ ". Selon mes recherches, la pratique de voler les légumes fut autrefois très répandue parmi les Acadiens de la Nouvelle-Écosse et, selon Anselme Chiasson, ce fut aussi un tour particulier aux Acadiens des Îles-de-la-Madeleine. Il ajoute que « la coutume populaire veut que ce ne soit pas un crime de voler les choux ce soir-là ${ }^{17} \gg$. Je peux moi-même confirmer la présence de la coutume dans le village de Pubnico-Ouest et quelques villages avoisinants. Pour ce qui est de la baie Sainte-Marie, Léonie Comeau inclut une belle description de l'activité dans son recueil My Acadian Heritage :

Le soir de l'Halloween, les enfants se vêtaient avec leurs habits les plus foncés pour ne pas être aperçus. Ils remplissaient leurs poches de navots, de choux et parfois de patates. C'était surtout des légumes ronds qui roulaient bien lorsqu'on les lançait. Les plus fanatiques charriaient leur stock dans des sacs à patates. Il s'agissait d'ouvrir la porte et de lancer un gros navot ou un chou dans la maison ; puis de s'enfuir à toutes jambes et de se cacher $[\ldots]$ tandis que le maître de la

14. CA, coll. Carmen d'Entremont, témoignage de M. Isaire d'Entremont (né le 22 septembre 1908), enregistré le 10 novembre 2004 à Pubnico-Ouest.

15. CA, coll. LABOR-d'Entremont, témoignage de $\mathrm{M}^{\mathrm{me}}$ Dolores-Ann d'Entremont (née le 12 octobre 1936), enregistré le 20 juillet 2006 à Pubnico-Ouest.

16. CA, coll. Labor-Dol, témoignage de $\mathrm{M}^{\mathrm{me}}$ Édith Comeau-Tufts (née le 2 juin 1919), enregistré le 20 juillet 2006 à Saulnierville.

17. Anselme Chiasson et al., "Le Folklore acadien », dans Jean Daigle (dir.), L'Acadie des Maritimes, Moncton, Université de Moncton, Chaire d'études acadiennes, 1993, p. 153. 
maison sortait pour voir qui était le coupable. Une vraie poursuite était terrifiante, mais amusante ${ }^{18}$.

Comme à la baie Sainte-Marie, on préférait les légumes ronds à Pubnico. Anselme Chiasson note qu'on volait des choux à Chéticamp la veille de la Toussaint pour les faire cuire le lendemain ${ }^{19}$. Une informatrice ajoute la remarque suivante : «Tu pouvais pas laisser des citrouilles ou des choux ou des navots dans le jardin, tu savais qu'à la Halloween ç'allait être volé. [...] ils alliont pirater ça qu'il y avait de reste dans le jardin ${ }^{20} »$. Ronald Labelle signale qu'à Chezzetcook aussi on avait coutume de voler des choux le 31 octobre : « les jeunes gens les arrachaient et en volaient quelques-uns pour jouer des tours aux propriétaires des jardins ${ }^{21} \gg$. Ici, on ne parle pas de soupes, mais, dans plusieurs cas, on explique qu'on prenait des légumes des champs avec l'intention de se faire une soupe aux choux.

Aujourd'hui, certains se divertissent toujours en jouant des tours durant la période de l'Halloween, mais les farceurs sont devenus moins innocents. $\mathrm{Au}$ lieu de voler les légumes pour les faire cuire, on en vole pour les détruire, comme le souligne une informatrice : « À cette heure, c'est les trafiquages de citrouilles. Ils venont par camions et ils défaisont les citrouilles dans le mitan du chemin ${ }^{22} \gg$. De plus, on a commencé, par exemple, à mettre le feu dans des maisons ou granges abandonnées, et dans les chemins, ou encore à lancer des objets après les véhicules la nuit, causant parfois des accidents assez graves, tandis qu'autrefois on prenait bien soin de ne pas endommager les biens. La mystification a certainement perdu son charme ainsi que son innocence à Pubnico-Ouest, mais certains tours datant du milieu du $\mathrm{xx}^{\mathrm{e}}$ siècle sont restés célèbres, y compris plusieurs vols de soupes, et ils sont toujours contés.

\section{Le vol des soupes aux choux}

Aussi, à Pubnico, les histoires sur le vol des soupes abondent, surtout parmi les aînés. En fait, que le vol soit réussi ou non, la majorité des gens nés pendant la première moitié $\mathrm{du} \mathrm{Xx}^{\mathrm{e}}$ siècle ont un récit comique à raconter sur une soupe volée. J'ai donc recueilli plus d'une douzaine de ces récits. Comme

18. Léonie Comeau-Poirier, My Acadian Heritage, Hantsport, Lancelot Press, 1985, p. 29 [traduction de l'auteur].

19. Anselme Chiasson, Chéticamp : Histoire et traditions acadiennes, Moncton, Éditions des Aboiteaux, 1961, p. 216.

20. CA, coll. Labor d'Entremont-Dol, $\mathrm{M}^{\mathrm{me}}$ Marie-Colombe Robichaud, le 19 juillet 2006.

21. Ronald Labelle, « La Vie acadienne à Chezzetcook, Nouvelle-Écosse », Les Cahiers de la Société historique acadienne, Moncton, La Société historique acadienne, vol. 22, n 2-3, avril-septembre 1991, p. 82 .

22. CA, coll. Carmen d'Entremont, $\mathrm{M}^{\mathrm{me}}$ Dolores-Ann d'Entremont, le 20 juillet 2006. 
plusieurs coutumes, il est difficile de déterminer où et quand tout a commencé. Néanmoins, la plus vieille attestation d'un vol de soupe que j'ai retrouvée remonte à 1886. Une jeune fille du milieu, Agnès-Clémentine d'Entremont, née le 11 décembre 1865, fait mention de la pratique dans un journal personnel. À plusieurs reprises, elle note qu'il y a eu une soupe chez un tel, et le 9 octobre 1886, elle donne l'entrée suivante: "There was a soup to Issac. David, Laundry [Landrie], Roger and Urbain went and stole the pot half full and came to Pius's [sic] to eat it. I had my share out of it. It was a pig $\operatorname{time}^{23} \gg$. Les témoignages recueillis dévoilent que la tradition fut en pleine vitalité au début $\mathrm{du} \mathrm{Xx}^{\mathrm{e}}$ siècle. Malheureusement, elle ne semble pas avoir dépassé le milieu du siècle ; elle aurait survécu au moins soixante ans.

Ce rituel d'automne se déroulait ordinairement en soirée, dans le cadre d'une veillée organisée. Les hôtes invitaient quelques amis à venir jouer aux cartes, chanter et, bien sûr, manger de la soupe. Puisque c'était une occasion pour les fréquentations, c'était la coutume d'y aller avec un partenaire, comme l'explique cette informatrice :

Ils faisiont des soupes, beaucoup, des soupes aux choux, à Hallowe'en. [...]. Oh oui, $[\ldots]$ ils faisiont un petit party puis là ils faisiont la soupe, puis ils mangiont de la soupe. Mais tu te trouvais manière d'un partenaire pour aller manger avec toi, manger la soupe, tu sais. Quelqu'un [disait] : « Eh bien, tu viendras manger de la soupe avec moi », tu sais. Ça là, c'était manière de drôle, but anyway, c'est de même c'était ${ }^{24}$.

En plus d'y aller pour fréquenter les gens et manger un repas, on y allait pour voir si la soupe serait volée, ce qui était souvent le cas selon les témoignages. Comme l'explique une autre informatrice : "Si elle était pas volée, c'était pas du fun du tout ${ }^{25}$ ». Après le vol, le jeu commençait puisque le but, selon plusieurs attestations, était d'emporter la soupe dans un autre lieu dans l'intention de la consommer avant d'être découvert, car les victimes circulaient souvent d'une maison à l'autre en espérant retrouver leur soupe. Il est intéressant de noter qu'une fois les voleurs rapportèrent à la fête la soupe volée : «Je crois qu'ils ont revenu avec la soupe pour point ruiner la soirée ${ }^{26}$ ».

Quelques-uns des témoignages révèlent que le vol était souvent exécuté lorsque la soupe était mise à refroidir dans les entrées ou les tambours. Si cela n'était pas le cas, on trouvait tout simplement une autre méthode : « Des fois, ils entriont et [...] ils creviont [éteignaient] les lampes et [...] ils voliont

23. CA, Fonds Agnès-Clémentine d'Entremont, MG 21, vol. II, 1886.

24. $\mathrm{CA}_{\mathrm{A}}$, coll. Carmen d'Entremont, $\mathrm{M}^{\mathrm{me}}$ Marjorie d'Entremont, le 4 janvier 2004.

25. CA, coll. LABOR-d'Entremont, témoignage de $M^{\text {me }}$ Lilian d'Entremont (née le 26 février 1919), enregistré le 12 juillet 2005 à Pubnico-Ouest-le-Centre.

26. Loc. cit. 
la soupe ${ }^{27} \gg$. Les joueurs de tours étaient créatifs, plus particulièrement ceux qui pouvaient entrer dans une maison remplie de gens sans qu'on s'en aperçoive. Certains ratoureux étaient si doués qu'ils réussissaient même à voler une soupe sur le poêle, comme c'est arrivé dans le cas suivant :

J'avons été voler des soupes. Première soupe, première année qu'ils avont fait de la soupe sur Delmar à Tim, là, qu'ils appelont, j'avons dit : «I guess faut j'allions la voler ». Et j'ons embarqué dans les cars et j'avons bouné [quitté]. Et tout le monde nous connaissait là. S'il y avait un party, ils alliont faire une Hallowe'en soup. Ils aviont une Hallowe'en soup sur le stove. Et j'avons rentré et j'avons starté à conter des yarns [histoires] avec la gang, you know. J'étions point invités, nous autres. Et Delbert à Louis à Martin était avec nous autres. Et la première chose, il a attrapé le pot et couri [couru] dehors. Le pot était gone. Jumper [sauter] dans la car, j'avons bouné sur Ralph, sur Poucha, là, manger la soupe $^{28}$.

D'autres mystificateurs avaient même bouché la cheminée d'une maison avec l'intention de faire boucaner le poêle en espérant faire sortir les filles pour pouvoir ensuite rentrer voler une soupe. Vers la fin d'octobre, les femmes faisaient la cuisine avec les yeux grand ouverts puisqu'il y avait toujours la menace de se faire voler un repas, surtout les célèbres soupes d'Halloween, comme l'explique une informatrice: «Et puis fallait qu'ils la watchirent parce [qu'il y] en avait d'autres à essayer à la voler pour aller à un[e] autre maison la manger ${ }^{29} »$.

La soupe aux choux, aussi connue sous les noms de Halloween soup et de cabbage soup, était assez ordinaire. Selon une informatrice, « il y avait presque rien dedans $\mathrm{s}^{30} »$. Le seul ingrédient spécial était le chou, et souvent c'était une soupe aux légumes qui était faite pour l'occasion. Selon quelques témoignages, on pouvait ajouter, entre autres, de la viande salée, des navots, des carottes, des patates et des féveroles. Comme l'explique une informatrice : « c'est rien que le temps de l'année qu'ils appelont ça des Halloween soups $^{31}$ ». Aujourd'hui, à Pubnico, plusieurs font toujours leur soupe aux choux de façon annuelle pour leur famille, comme aussi les Dames auxiliaires des pompiers de Pubnico-Ouest qui font une grosse soupe aux choux communautaire vers le 31 octobre.

$$
* * *
$$

27. CA, coll. LABOR-d'Entremont-Dol, témoignage de $M^{\text {me }}$ Anne d'Entremont (née le 2 mai 1937), enregistré le 3 août 2006 à Pubnico-Ouest-le-Centre.

28. CA, coll. Carmen d'Entremont, M. Florent d'Entremont, le 7 février 2005.

29. CA, coll. Carmen d'Entremont, témoignage de $\mathrm{M}^{\mathrm{me}}$ Annette Pothier-Comeau, enregistré le 27 juillet 2006 à Pubnico-Ouest-le-Centre.

30. CA, coll. Carmen d'Entremont, $\mathrm{M}^{\mathrm{me}}$ Lilian d'Entremont, le 12 juillet 2005.

31. CA, coll. Carmen d'Entremont, $\mathrm{M}^{\mathrm{me}}$ Marjorie d'Entremont, le 24 novembre 2004. 
Ainsi, si le vol des légumes s'étend parmi les Acadiens de la NouvelleÉcosse et jusqu'aux Îles-de-la-Madeleine, le vol des soupes aux choux serait plutôt une pratique limitée à la région de Pubnico-Ouest et de quelques villages avoisinants. Ce qui est surtout frappant, c'est la grande vitalité que cette tradition a connue jusqu'au milieu du $\mathrm{xx}^{\mathrm{e}}$ siècle. Depuis les premières attestations relevées dans mon mémoire de maîtrise, de nouvelles informations ont fait surface. Tout récemment, un Acadien du village de Wedgeport certifiait l'existence de la pratique dans son milieu. Il semble que le moment est venu d'élargir cette recherche, de l'étendre à toute l'Acadie et même au delà. Voilà la raison de cette note de recherche, qui s'attache au patrimoine oral d'un groupe ancien, mais souvent oublié dans l'étude du folklore acadien. 